Annuaire suisse de politique de développement

11 | 1992

Annuaire Suisse - Tiers Monde 1992

\title{
La CNUED, les enjeux pour la Suisse
}

Alain Clerc

\section{OpenEdition}

\section{Journals}

Édition électronique

URL : http://journals.openedition.org/aspd/1565

DOI : $10.4000 /$ aspd. 1565

ISSN : 1663-9669

\section{Éditeur}

Institut de hautes études internationales et du développement

\section{Édition imprimée}

Date de publication : 1 janvier 1992

Pagination : 205-212

ISSN : 1660-5934

\section{Référence électronique}

Alain Clerc, "La CNUED, les enjeux pour la Suisse », Annuaire suisse de politique de développement [En ligne], 11 | 1992, mis en ligne le 18 mai 2013, consulté le 08 septembre 2020. URL : http:// journals.openedition.org/aspd/1565; DOI : https://doi.org/10.4000/aspd.1565 


\title{
La CNUED, les enjeux pour la Suisse
}

\author{
Alain Clerc
}

\section{Pourquol la CNUED}

La terre a mal à son environnement. Cela seul justifie-t-il une conférence? Pour certains le motif est suffisant, pour d'autres, il n'est pas opportun de réunir, à grands frais, une conférence qui n'ira guère au-delà d'un constat de l'état déplorable de notre environnement. C'est dans ce contexte qu'il convient de s'interroger sur les raisons de cette conférence à la lumière des intentions de ses initiateurs.

L'idée de la CNUED émane d'un petit groupe de spécialistes des questions environnementales qui souhaitent adapter aux exigences nouvelles les structures des institutions qui gouvernent depuis vingt ans sur le plan national, régional et international les questions de l'environnement. A côté d'une série de questions qui nécessitent un engagement accru de la communauté internationale (désertification, préservation de la forêt tropicale, changements climatiques, gestion de l'eau, etc.), l'enjeu de la CNUED est donc d'abord institutionnel.

Dans divers domaines, il apparaît en effet souhaitable de se doter de nouveaux moyens d'action pour répondre aux urgentes nécessités d'une situation qui à bien des égards est dramatique et qui ne cesse de se détériorer. A défaut d'accords ou d'engagements concrets et contraignants, la CNUED doit s'entendre sur les termes et les délais de la mise en place des structures nécessaires à l'adoption de ces accords. Pour aboutir de tels résultats, il convient de quantifier les ressources qu'exige la solution de chacun de ces objectifs. C'est ainsi que l'on procède dans tout projet d'investissement.

\section{Les dérapages}

Ce processus, ambitieux en lui-même, découle des propositions contenues dans le rapport "Notre avenir à tous" (Our common futur) de la Commission Environnement et Développement. Cette Commission avait été mise en place à l'initiative des représentants de la Suisse, de la Suède, du Canada et du Japon au début 
des années 80 . Les négociateurs traditionnels des questions environnementales sont tout désignés pour engager ce débat. lls connaissent les faiblesses et les limites des institutions actuelles, ainsi que les obstacles à l'adoption de nouveaux accords partiels ou globaux sur les principales questions de l'environnement. Malheureusement, ces acteurs ont été partiellement dessaisis du dossier lorsque, sous l'influence convergente d'intérêts divergents, le dossier de la CNUED fut confié à l'Assemblée Générale, un organe essentiellement politique, certes capable de mettre sur pied la plus grande conférence de l'environnement jamais organisée sur notre terre mais guère motivé par la dialectique environnementale.

La lourde machinerie établie pour gérer le processus de la préparation de la conférence, contrairement au concept adopté pour la Commission Brundtland a d'ailleurs été critiquée par certains détracteurs de la CNUED qui l'ont même qualifié de véritable chape bureaucratique et diplomatique sur une idée généreuse. Un comité préparatoire comprenant des représentants de tous les pays membres des Nations Unies et du PNUE doit en effet se réunir des semaines durant pour plancher sur une myriade de questions dans trois groupes de travail et de nombreux sous-groupes. La règle du consensus prévaut. II n'y a pas non plus d'objectifs précis, d'engagements contraignants mais un vaste programme de bonnes intentions pour replâtrer d'ici l'an 2000 et au-delà ce qui peut l'être. Stockholm, vingt ans plus tôt, avait donné jour à une vingtaine de principes qui constituent encore les fondements de l'action environnementale. Sans mettre en cause ce qui est devenu avec le temps des règles de droit international, ce catalogue de principes aurait pu être complété sur des aspects nouveaux. Cet objectif n'a pas été retenu.

Aucun effort n'a été entrepris non plus pour quantifier sérieusement l'effort financier que la communauté internationale et en particulier les pays de l'OCDE doivent consentir aujourd'hui pour répondre aux principaux défis de l'environnement d'ici l'an 2000 et au-delà. Sans ces données, toute action ou engagement relève de la pétition de principe et ne répond pas à l'expectative des pays en développement.

A un moment où l'environnement figure sur tous les agendas et qu'une majeure partie des communiqués de presse des principales manifestations politiques s'y réfèrent, on constate une surprenante absence de volonté politique pour que le projet de la CNUED aboutisse à des résultats tangibles. Pourtant un échec à Rio de Janeiro sera un échec à la fois pour les Nations Unies, pour le PNUE et pour tous ceux qui croient à la nécessité d'une nouvelle solidarité internationale pour assurer un autre développement au début du prochain millénaire.

\section{La Suisse et la CNUED}

Notre pays, dans cette conjoncture, n'a de prime abord guère de chance de modifier le cours des choses. Tenu à l'écart des Nations Unies par la volonté populaire, relégué au titre d'observateur à l'Assemblée Générale, ses moyens d'intervention sont apparemment limités. Si cette conception prévaut, la délégation Suisse joindra sa voix à Rio de Janeiro aux proclamations de bonne volonté, elle apportera sa contribution au débat et reviendra au pays avec le sentiment d'avoir parti- 
cipé à un grand moment de l'histoire du monde. Certains milieux partagent cette attitude. Cette approche a l'apparence du réalisme. Elle s'inscrit cependant dans une perspective frileuse, dans une vision sans ambition de notre politique étrangère et dans la condamnation "de facto" du róle des Nations Unies, comme enceinte privilégiée de gestion des questions environnementales.

La CNUED, indépendamment d'un échec ou d'un succès, représente pourtant pour notre pays une chance inespérée de revaloriser notre politique extérieure, et vraisemblablement une des dernières occasions d'assumer des responsabilités internationales. Sous l'impulsion de l'ancien Secrétaire d'Etat aux Affaires Etrangères Edouard Brunner, notre pays avait tenu un tel rôle dans les années soixante-dix dans le cadre plus restreint de la Conférence sur la sécurité et la coopération en Europe (CSCE). En dépit de notre neutralité, nous fûmes alors à la pointe d'une action internationale qui contribua aux changements profonds qui bouleversent aujourd'hui encore le paysage européen. La Suisse, les pays de l'AELE et les Neutres n'ont pas su au lendemain du départ de $M$. Edouard Brunner, tirer profit de cet effort et se sont marginalisés. L'engagement d'un homme dans un contexte difficile nous avait permis de contribuer à l'émergence d'une Europe nouvelle. Sous l'impulsion d'un autre homme, le Conseiller fédéral Flavio Cotti, la Suisse a pris depuis plusieurs années une place de marque dans les questions environnementales. Notre politique interne de l'environnement comme notre action internationale constituent des références pour le reste du monde. II serait regrettable de ne pas tirer profit d'un tel capital.

Comme dans tous les domaines, la mise en place d'une nouvelle politique nécessite un effort de longue haleine, une stratégie qui ne laisse aucune place aux atermoiements. Si le Président de la Confédération, en cette année du 700ème, a maintes fois réitéré sa volonté d'agir, certains milieux paraissent guère empressés de poursuivre l'action entreprise. Rio de Janeiro représente pourtant un forum inespéré. Notre pays a un triple intérêt à s'engager avec détermination dans la CNUED, à se démarquer par des initiatives propres et à apporter un appui matériel, logistique et politique au Programme des Nations Unies pour l'environnement en vue d'assurer son renforcement: un intérêt économique, politique et institutionnel.

\section{Premièrement un intérét économique}

Notre réglementation plus sévère stimule l'innovation et l'imagination et aiguise notre capacité de concurrence. Des produits, des services sont mis sur le marché qui doivent nous créer des débouchés au fur et à mesure que la fibre environnementale touche d'autres pays. En relayant cet effort de sensibilisation, notre diplomatie peut contribuer à asseoir notre présence sur ces nouveaux marchés. Les possibilités d'actions dans ce domaine sont innombrables et peu exploitées. Le déploiement d'une "diplomatie verte" à l'étranger favoriserait notre expansion économique. Notre politique extérieure marquée par des réflexes trop traditionnels semble pourtant hésiter encore à s'engager sur cette voie.

La préparation de la Conférence de Rio de Janeiro nous fournit l'occasion de revoir ces conceptions. Dans tous les continents et dans plusieurs capitales, des 
postes de conseillers environnementaux pourraient, par exemple, être créés, et cela simplement par la reconversion d'activités diplomatiques ou para-diplomatiques tombées en désuétude. Mais, au-delà de cet aspect diplomatico-commercial notre intérêt économique se situe dans la reformulation de notre politique d'investissement à moyen et long terme. L'économique est une des activités humaines qui s'est développée sans contrainte dans la conception d'un monde infini aux ressources inépuisables. Ce schéma de pensée est caduc. Notre effort d'imagination doit se concentrer sur d'autres modes de fonctionnement des mécanismes économiques. Les conditions de notre développement doivent être repensées en fonction d'un monde ou l'économique est soumis aux contingences contraignantes des limites de notre biosphère. Cet effort d'imagination doit être stimulé dans tous les pays. Le tournant du siècle sera marqué par une approche différente des questions économiques. Notre pays dispose de chercheurs, d'ingénieurs-conseils et d'économistes de talent déjà partiellement confrontés à ces problèmes. Leurs efforts canalisés vers ces nouveaux objectifs bénéficieront à l'ensemble de notre économie. Le créneau environnemental constituera vraisemblablement le moteur de l'activité économique au début du siècle prochain et cela mẻme dans la perspective d'une économie de plus en plus confinée à un concept de survivance. II ne s'agit pas, cependant, de simplement développer un "éco-business" (qui trahit les principes de l'écologie), il s'agit d'envisager les alternances acceptables à des modes de développement dépassés. C'est dans cette voie que la nouvelle Académie de l'environnement de Genève doit envisager son action, à la fois sur le plan interne et externe. On jugera du succès de cette nouvelle "environnemental school" à sa capacité de faire école et d'engager les innombrables "business school" à changer de caractère. Les milieux économiques se mobilisent et les initiatives de M. Stephan Schmidheiny vont "grosso modo" dans le même sens. La création de la "swatchmobil" et le développement à Genève d'une entreprise telle qu'»Ecosphère", qui veut concrètement répondre aux besoins spécifiques du tiers monde dans la domaine de l'environnement, en mobilisant les meilleures compétences techniques et financières de nos entreprises, sont des mesures qui relèvent déjà de ce nouvel esprit économique. La préparation de la CNUED nous offre donc la chance de réfléchir à notre destin économique et au rôle de notre économie dans un monde en mutation écologique.

En raison d'un éveil écologique plus prononcé que d'autres pays nous disposons d'un avantage stratégique. De nombreuses institutions académiques, nongouvernementales, voire financières peuvent contribuer à développer cette réflexion. Notre pays pourrait ainsi utiliser l'avant et l'après Rio de Janeiro pour esquisser les contours d'une nouvelle approche économique et tenter quelques propositions courageuses (par exemple au niveau de l'adoption de taxe ou de «users fees"). Au moment d'âpres discussions sur le rôle de l'Etat, sur le redimensionnement de certaines industries (notamment dans le complexe militaro-industriel), à la veille d'une reprise économique qui se fait attendre, en période d'innombrables faillites, une réflexion approfondie sur les interfaces de l'environnement et de l'économie pourrait s'avérer particulièrement salutaire pour notre pays et pour ses institutions. 


\section{Deuxièmement un intérêt politique}

Nord-Sud et pour réorienter certaines négociations économiques actuellement dans l'impasse. Pour atteindre cet objectif, les diplomates doivent impérativement renoncer à intégrer la réflexion environnementale dans un concept traditionnel de négociation, comme ils sont tentés de le faire. Ils doivent chercher d'autres voies. Si les négociateurs environnementaux sont conscients de cette exigence, notre "diplomatie politique" ne semble pas prête encore à jouer la carte environnementale.

Or la CNUED, par le simple fait qu'elle existe, nous interpelle sur les nouvelles structures à créer pour intégrer l'environnement dans nos schémas politiques. Sur le plan intérieur, nous devrons adopter nos structures politiques qui ne tiennent pas compte de l'interdisciplinarité des questions environnementales. La Confédération s'est dotée d'un Office fédéral de l'environnement, des forêts et du paysage, de nombreux cantons ont conçu des postes de "coordinateur de l'environnement", mais au delà de ces réponses traditionnelles qui correspondent aux exigences de l'environnement tel qu'on les concevait il y a vingt ans, aucune réflexion politique n'a été engagée pour intégrer l'environnement dans notre philosophie politique. Reflets d'un manque de perspicacité, d'un manque de courage, peur du changement ou tout simplement incrédulité face à un mouvement qui surprend, le phénomène vert n'a pas ébranlé notre réflexion et reste encore sans effet sur nos institutions. Les postulats écologiques nous sollicitent en pleine récession politique, à un moment où les principes du matérialisme socialiste s'écroulent et les lois du "laissez-faire" semblent redevenir le seul credo de la pensée politique. Si le "management socialiste" fut catastrophique pour la gestion de l'environnement, nous nous leurrerions en méconnaissant les "méfaits" du libéralisme sur notre environnement. En panne de doctrine, aucun mouvement politique n'apparait toutefois en mesure de proposer aujourd'hui une alternative.

Michel Serres et d'autres philosophes évoquent la nécessité d'un nouveau "contrat naturel". La gent politique acquiesce en feignant de croire qu'un peu plus d'environnement dans les mécanismes économiques résoudra les problèmes. L'écologie, à la veille du deuxième millénaire, représente un tout autre défi, celui d'une nouvelle conception des rapports humains et des rapports de l'homme avec la nature dans un monde désormais fini avec des ressources naturelles qui ne sont plus la propriété privée des seuls Etats mais qui servent les intérêts de tous. Les événements qui secouent l'empire soviétique et mettent également en cause le concept d'Etat-nation doivent être envisagés dans cette même optique. II n'est d'ailleurs pas sans intérêt de noter à ce propos que les forces politiques qui ont renversé les pouvoirs communistes dans les pays de l'Est étaient souvent imprégnées de sentiments écologiques.

L'écologie va donc aujourd'hui remettre en cause une conception traditionnelle de l'Etat qui s'oppose aux postulats de la nature. L'émergence de nouvelles instances interétatiques au niveau régional est d'ailleurs assez significative de cette évolution. Des accords tels que ceux qui lient les départements de l'Ain, de la Haute-Savoie et les cantons du Valais, Vaud et Genève, sont des organisations qui d'une certaine manière remettent en cause la notion d'Etat-nation. Dans tous 
ces accords l'environnement joue un rôle moteur. Dans le domaine sensible des ressources énergétiques, les puissances économiques qui dépendent de ces ressources n'ont pas hésité à conjuguer leurs intérêts et même à intervenir militairement contre des Etats qui à un titre ou à un autre mettaient en cause leurs intérêts économiques. Ces démarches ont aussi concouru à relativiser la souveraineté des Etats sur leurs ressources naturelles. II ne faut pas, cependant, s'attendre à ce que la Conférence de Rio de Janeiro remette en cause le principe 21 de la Convention de Stockholm relatif à la souveraineté des Etats sur leurs ressources nouvelles ou qu'elle exhorte les pays à une gestion décentralisée des questions environnementales. La préparation de la CNUED constitue néanmoins une occasion unique de s'interroger et de réfléchir sur ces questions. Notre pays doit se donner les moyens de cette réflexion. II est souhaitable qu'il contribue à la conférence par le développement d'idées originales et cela même si cette réflexion doit aboutir à la remise en cause de certains tabous politiques.

\section{Un intérêt institutionnel}

En dépit d'une réglementation environnementale très développée résultant d'une chimérique volonté politique de colmater toutes les fissures provoquées par une évolution économique incontrôlée, les réponses juridiques dont nous disposons sont terriblement inadaptées. Le processus de réflexion politique doit donc conduire soit au renforcement des institutions existantes soit à leur remplacement par de nouvelles structures.

Les paramètres sont connus, les problèmes sont interdisciplinaires (étroitement liés les uns aux autres dans leurs causes et leurs effets), ils sont ponctuels et globaux, ils affectent l'approche micro- et macro-économique de notre développement, ils sont liés à l'évolution démographique dans un monde fini aux ressources limitées, ils s'additionnent dans leurs effets, ils ont un impact économique (financier et commercial), social, politique et culturel, ils sont la source de conflits de plus en plus fréquents.

Que ce soit au niveau des administrations communales, cantonales ou fédérales, les décisions sont adoptées par une multitude d'organes sans qu'elles soient examinées dans une perspective écologique. L'institution d'un examen écologique de l'ensemble des décisions qui affectent notre développement devient dés las incontournable. Au même titre que nous avons un Monsieur Prix, nous devrions disposer d'un Monsieur Environnement qui pourrait agir comme instance de recours. Sur le plan parlementaire également, l'ensemble des décisions politiques, sociales et économiques devrait être ventilés en fonction de leur impact écologique. Les projets de l'administration devraient être élaborés en étroite concertation avec les acteurs concernés du secteur privé et des organisations écologiques. La mise en place d'une Agence de l'environnement dotée de pouvoirs contraignants dirigée par un triumvirat reflétant les intérêts distincts des principaux partenaires sociaux devient une nécessité. La mise en place d'une institution similaire à la Banque nationale a déjà été évoquée et pourrait constituer,mutatis mutandis,un modèle intéressant. 
Le Chef du Département de l'Intérieur flanqué d'un super Secrétaire d'Etat veillerait à la fois à coordonner l'ensemble des décisions politiques et économiques qui ont un impact sur l'environnement et à dessiner les grandes orientations de notre politique nationale de l'environnement en s'assurant de sa conformité avec les objectifs de la politique européenne et globale.

L'Agence ou les agences (si l'on désire développer une action qui tiennent compte des diverses configurations géographiques et géologiques de notre pays) devraient développer un système d'observation en continu de notre environnement dans le cadre d'un «monitoring" environnemental général de notre planète. Notre comptabilité nationale devrait refléter (comme cela existe déjà dans d'autres pays) les coûts résultant de la gestion de notre environnement. On pourrait enfin envisager une institution d'arbitrage (Conseil des Sages) qui s'efforcerait de trancher les inévitables litiges.

Ce catalogue de mesures n'est de loin pas exhaustif, il reflète quelques thèmes centraux de la réflexion institutionnel que nous ne pouvons plus esquiver. La Conférence de Rio laborieusement préparée au niveau des fonctionnaires n'aboutira à des résultats que si les pouvoirs politiques assument leur responsabilité. L'action internationale doit être mûrie à la lumière d'expériences locales ou nationales. Les enjeux de la CNUED passent donc nécessairement pas un réexamen de notre politique nationale. II faut donc espérer que notre pays participe au premier "Earth Summit" avec quelques idées novatrices. Notre position privilégiée, favorise une action de pointe de notre pays l'année prochaine.

Sur le plan international, notre pays s'est déjà fait remarquer par une multitude d'initiatives engagées d'abord par $\mathrm{M}$. Egli et développées ensuite avec force par M. Cotti (établissement du GRID, de la Commission Brundtland, du Secrétariat de la Conférence de Rio de janeiro, du Secrétariat de la Convention de Bâle à Genève, présidence de la Conférence de Bâle sur le mouvement des déchets dangereux, de la deuxième Conférence sur le climat, mise en place d'un organe de concertation AELE-CE, initiative concernant le protocole sur les composés sulfureux et les oxydes d'azote, convocation d'une deuxième Conférence environnement Est-Ouest, initiative et financement du Bureau d'information sur le climat, lancement d'un projet de Croix Rouge de l'environnement, etc.).

Ce catalogue d'actions internationales nous impose d'agir à Rio de Janeiro avec de nouvelles idées. A vrai dire, une attitude de repli à la CNUED au premier sommet des chefs d'Etats de notre planète paraîtrait incompréhensible. Notre action pourrait se dérouler autour de cinq axes. Elle aurait pour corollaire un renforcement du Programme des Nations Unies pour l'environnement et devrait tendre à renforcer le rôle de la Suisse et de Genève dans la négociation internationale. Premièrement, l'établissement d'un "Centre mondial d'intervention et d'assistance en cas d'accidents" en étroite symbiose avec la Croix-Rouge et en tirant profit des infrastructures déjà disponibles; deuxièmement, le renforcement de la surveillance environnementale ( $y$ compris l'inspection et l'arbitrage; troisièmement, la formation avec le renforcement de l'Académie internationale de Genève, notamment en soutenant les propositions du Directeur exécutif du PNUE, Monsieur Mostafa K. Tolba, en faveur d'une Académie mondiale de l'environnement; qua- 
trièmement, le renforcement des organes de coordination au sein de système des Nations Unies en favorisant l'établissement à Genève d'un véritable organe de coordination et, le cas échéant, d'une commission spéciale de l'Assemblée générale pour les questions environnementales; cinquièmement, le renforcement du Conseil de Sécurité qui pourrait se doter d'un organe prêt à intervenir en cas de menaces environnementales ou de graves litiges écologiques.

Ces mesures de nature globale se superposent aux mesures qu'il convient d'engager sur le plan national, leur mise en oeuvre sur le plan international ne seraient pas sans impact sur notre politique nationale. Ces actions s'inscrivent dans le continuité de notre engagement intemational. Elles seraient à la fois l'expression de notre solidarité internationale et de notre volonté d'oeuvrer pour une meilleure protection de notre environnement. Elles s'inscriraient dans une volonté clairement établie d'assurer le maintien et le développement d'une politique suisse progressiste dans le domaine de l'environnement. Elles nécessiteraient un engagement accru de la Confédération dans l'une des enceintes les plus actives des Nations Unies, le PNUE, dont le siège est à Nairobi. Elles n'exclueraient pas une composante régionale et européenne dans le mesure ou notre pays, en renforçant le rôle global du PNUE, pourrait parallèlement s'engager à développer son rôle régional. Les événements qui secouent l'Europe de l'Est nous obligent à réexaminer aussi notre rôle dans ce contexte. Une initiative visant à établir à Genève une Agence de l'environnement de l'ensemble des pays européens, étroitement liée et soutenue par le PNUE et tirant profit des équipements déjà disponibles à Genève pourrait également contribuer à l'essor de notre région considérée comme un sous-ensemble de notre planète.

\section{Conclusion}

La CNUED marquera, nolens, volens, notre histoire. Ceux qui refusent de croire que notre monde est en mutation ne verront dans cette manifestation qu'un exercice diplomatique. Un de plus. Ceux qui appréhendent les changements minimiseront la portée de l'événement. lls seront confortés dans cette attitude par tous les hommes, notamment dans les pays en développement, en butte avec un immédiat trop cruel pour apprécier la gravité du "mal-développement". Ceux qui, peu concernés sur le fond, cherchent essentiellement un succès médiatique utiliseront la Conférence à des fins politiciennes. L'ensemble de ces forces risque dès lors de se conjuguer pour que Rio de Janeiro soit un événement sans effet. C'est le risque de toute conférence et ses promoteurs ne l'ignorent pas. Mais Rio de Janeiro, c'est aussi un jalon dans une réflexion environnementale qui mûrit depuis vingt ans. C'est dans ce processus de réflexion que nous devons nous situer. C'est à ce niveau que notre contribution constitue une chance de façonner un monde différent de celui qu'autrement nous risquons de laisser, faute d'imagination et de perspicacité, aux générations futures. C'est à ce titre que l'histoire se souviendra de Rio de Janeiro et peut-être de la contribution suisse. 